\title{
Transcription factors and genetic circuits orchestrating the complex, multilayered response of Clostridium acetobutylicum to butanol and butyrate stress
}

Qinghua Wang ${ }^{1,2,3 \dagger}$, Keerthi Prasad Venkataramanan ${ }^{1,4 \dagger}$, Hongzhan Huang ${ }^{1,2,3}$, Eleftherios T Papoutsakis ${ }^{1,4^{*}}$ and Cathy $\mathrm{H} \mathrm{Wu}^{1,2,3^{*}}$

\begin{abstract}
Background: Organisms of the genus Clostridium are Gram-positive endospore formers of great importance to the carbon cycle, human normo- and pathophysiology, but also in biofuel and biorefinery applications. Exposure of Clostridium organisms to chemical and in particular toxic metabolite stress is ubiquitous in both natural (such as in the human microbiome) and engineered environments, engaging both the general stress response as well as specialized programs. Yet, despite its fundamental and applied significance, it remains largely unexplored at the systems level.

Results: We generated a total of 96 individual sets of microarray data examining the transcriptional changes in $C$. acetobutylicum, a model Clostridium organism, in response to three levels of chemical stress from the native metabolites, butanol and butyrate. We identified 164 significantly differentially expressed transcriptional regulators and detailed the cellular programs associated with general and stressor-specific responses, many previously unexplored. Pattern-based, comparative genomic analyses enabled us, for the first time, to construct a detailed picture of the genetic circuitry underlying the stress response. Notably, a list of the regulons and DNA binding motifs of the stress-related transcription factors were identified: two heat-shock response regulators, HrcA and CtsR; the SOS response regulator LexA; the redox sensor Rex; and the peroxide sensor PerR. Moreover, several transcriptional regulators controlling stress-responsive amino acid and purine metabolism and their regulons were also identified, including ArgR (arginine biosynthesis and catabolism regulator), HisR (histidine biosynthesis regulator), CymR (cysteine metabolism repressor) and PurR (purine metabolism repressor).

(Continued on next page)
\end{abstract}

\footnotetext{
* Correspondence: papoutsakis@dbi.udel.edu; wuc@dbi.udel.edu

${ }^{\dagger}$ Equal contributors

'Delaware Biotechnology Institute, University of Delaware, Newark, DE 19711,

USA

${ }^{2}$ Center for Bioinformatics and Computational Biology, University of

Delaware, Newark, DE 19711, USA

Full list of author information is available at the end of the article
} 
(Continued from previous page)

Conclusions: Using an exceptionally large set of temporal transcriptional data and regulon analyses, we successfully built a STRING-based stress response network model integrating important players for the general and specialized metabolite stress response in C. acetobutylicum. Since the majority of the transcription factors and their target genes are highly conserved in other organisms of the Clostridium genus, this network would be largely applicable to other Clostridium organisms. The network informs the molecular basis of Clostridium responses to toxic metabolites in natural ecosystems and the microbiome, and will facilitate the construction of genome-scale models with added regulatory-network dimensions to guide the development of tolerant strains.

Keywords: Gene expression, Protein-protein interaction, Transcriptional regulatory network (TRN), Transcription factor (TF), TF binding site (TFBS), Transcriptional regulator (TR)

\section{Background}

Clostridium organisms are endospore-forming anaerobic firmicutes important in pathogenesis, human physiology (with their notable role in the human gut microbiome [1]), the carbon cycle and biotechnological applications [2,3]. C. acetobutylicum is the first sequenced Clostridium and has evolved into a model organism for the genus. It can utilize a wide variety of substrates to produce metabolites useful as industrial chemicals and biofuels [4]. Most of these metabolites, and notably butyrate and butanol, are toxic to the cells greatly impacting their metabolism and survival [5]. Several studies have been published in the last few years aiming to understand the transcriptional and translational basis of metabolite stress response, yet the regulatory network beneath these responses remains incompletely understood at the systems level [6-14]. What transpires from the data of these studies is that the metabolite stress response includes virtually all annotated genes of the core stress program (the so-called heat-shock protein (HSP) response) but also many other large programs, including amino-acid and nucleic-acid biosynthetic pathways. Such programs are apparently underlying the adaptive response of the cells to these toxic metabolites. This adaptive response includes changes in membrane composition, such as increasing the fatty acid tail length and percentage of saturated fatty acids, which is known as homeoviscous adaptation in response to the fluidizing effects of organic solvents and acids [15-17].

Many bacterial genome sequences have been completed and successfully annotated, but most lack information on the regulatory front of gene expression [18]. Understanding the complex regulatory circuitry comprised of transcription factors (TFs) and their corresponding DNA targets, including the motifs or transcription factor binding sites (TFBSs), is a fundamental requirement for understanding the complexity of responses in natural habitats and microbiomes, but also for building systems-level molecular models. Integrated use of detailed experimental data and in silico analyses is necessary for reconstructing transcriptional regulatory networks (TRNs) [19], which improve our understanding of complex phenotypes and facilitate the development of novel strains using synthetic biology.
In this study, we applied a comparative-genomics, pattern-based approach to analyze a deep set of temporal transcriptional data to infer the transcriptional regulatory interactions underlying the metabolite stress response in $C$. acetobutylicum (Figure 1). Using Regulatory Sequence Analysis Tools (RSAT) [20], footprint-discovery [21] analysis (i.e., phylogenetic footprinting) was carried out, where the comparative analysis of the genomic context of C. acetobutylicum genes was successfully accomplished. Overrepresented oligonucleotides (words) or spaced pairs thereof (dyads) were detected and assembled into Position Weight Matrices (PWMs) [20,21]. These matrices were compared against the PWMs of known TF binding DNA motifs from three public resources: RegPrecise [22], RegTransbase [23] and PRODORIC databases [24] with the tool Tomtom [25]. The results, together with OMA (Orthologous MAtrix) orthology inference in C. acetobutylicum for the genes available from RegPrecise [22,26], enabled the prediction of many TF binding sites (TFBSs) and functional assignment of select TFs and their target genes (TGs) in $C$. acetobutylicum (Figure 1). Together, results from these analyses enabled the construction of the stress response network involving the regulatory connections of key TFs and TGs engaged during stress.

First, we identify the common and distinct cellular responses to butanol and butyrate stress based on our comprehensive set of temporal transcriptional data. For the common stress responses, facilitated by target-gene predictions for the core HSP TFs, HrcA and CtsR, we expand the list of Class I, III \& IV stress genes. For the specialized stress responses, we identify the genes and biological processes that display distinct expression patterns for each metabolite. This, coupled by a genomescale analysis of transcriptional regulators (TRs) differentially expressed under the two stressors, led us to focus on core TFs that apparently orchestrate these stress responses. We analyzed the TFBSs and regulons of these TFs and examined the interconnectivity of their regulons thus arriving at a comprehensive picture of the transcriptional network that underlies the responses to two metabolite stresses. 


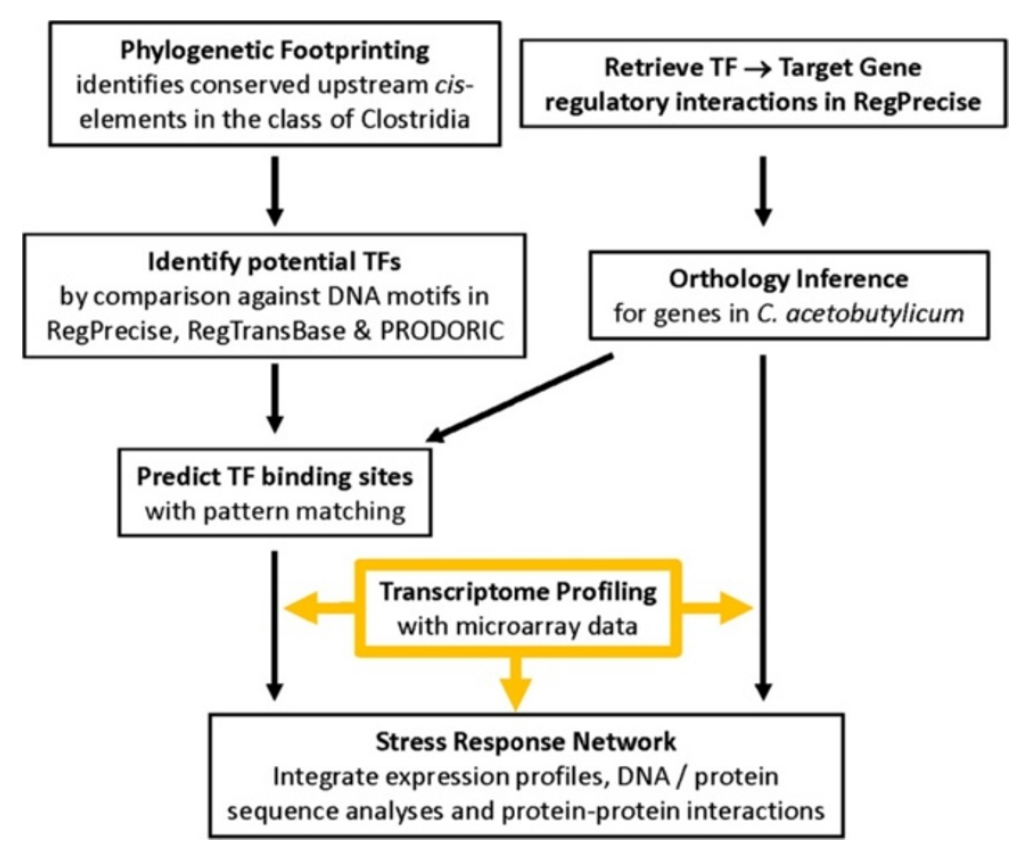

Figure 1 Workflow for prediction of transcriptional regulatory interactions to reconstruct the metabolite stress response network in C. acetobutylicum.

\section{Results and discussion}

Overview: systems analysis identifies common and distinct cellular responses to butanol and butyrate stress We use microarray analysis to examine the response of $C$. acetobutylicum to butanol and butyrate stress at midexponential phase $\left(\mathrm{A}_{600} \sim 1.0\right)$ of $\mathrm{pH}$-controlled batch cultures up to $75 \mathrm{~min}$ post stress with $15 \mathrm{~min}$ intervals $(0,15$, 30, 45, 60 and $75 \mathrm{~min})$. For each metabolite, cultures were stressed with three different concentrations: $30 \mathrm{mM}$ (low), $60 \mathrm{mM}$ (medium) and $90 \mathrm{mM}$ (high) of butanol, or with $30 \mathrm{mM}$ (low), $40 \mathrm{mM}$ (medium) and $50 \mathrm{mM}$ (high) of butyrate. These stressor concentrations were selected based on previous studies $[6,7,10]$ to obtain weak, moderate and strong stress responses. As described in the Methods section, we used a reference design to collect the data. Normalized microarray data were analyzed using significance analysis of microarrays [27] (SAM) to identify differentially expressed genes. We collected data from two biological replicates, with dye swaps for each of the 6 time points for each of the six stress conditions (butanol, butyrate; low, medium and high stress). Thus, we generated a total of 96 individual sets of stress data for each metabolite. These data constitute an extensive microarray data ensemble capturing the response of $C$. acetobutylicum to these two metabolites. The design of the microarrays based on the Agilent technology and the associated methods have been extensively validated $[5,28]$. This microarray technology offers extraordinary accuracy with many probes for each gene and multiple copies of each probe. Nevertheless, the expression data for a select subset of the genes (CAP0102, CAC1405,
CAC3190, CAC0766 and CAC1391; see Methods section for details) were further validated using Q-RT-PCR from a third biological replicate.

Consistent with an earlier, lower resolution microarray study $[6,7,10]$, our data show that butanol and butyrate stress responses of $C$. acetobutylicum include not only upregulation of heat shock protein (HSP) genes, but also the differential expression of more than 1,000 genes related to many distinct physiological functions and cellular programs (Additional file 1). Under butanol stress, 1,118 genes were differentially expressed (535 up, 575 down, 8 bimodal); whereas there are 1,390 differentially expressed genes for butyrate stress (710 up, 675 down, 5 bimodal). The union of the two includes 1,984 genes, for which K-means clustering results are shown for the butanol and butyrate stress (Additional file 2: Figure S1A and B), respectively.

Comparative analysis of the butanol $(\mathrm{BuOH})$ versus butyrate (BA) stress responses is essential for understanding the general (that is, the common) stress response as well as the specialized, stressor-dependent responses. Using FIVA (Functional Information Viewer and Analyzer) [29], we identified the statistically significant differentially expressed functional categories based on annotated pathways (KEGG database [30]) and Gene Ontology (GO) annotations (from UniProtKB [31]) for the $C$. acetobutylicum genome. Each gene was assigned into one of four differentially expressed groups (up-regulation, down-regulation, bimodal and non-significant) for each stressor individually or in combination (e.g., $\mathrm{BuOH}$-up/BA-up, BuOH-down/BA-down, BuOH-up/ 
BA-down etc.). Then, the GO categories and the KEGG pathway classifications for each gene were utilized in FIVA to identify the significant functional categories enriched for each differentially expressed group. In Figure 2, the Venn diagrams display the most significant similarities and differences between the butanol and butyrate stresses.
The common \& general stress response: expanding the list of Class I, III \& IV genes

Consistent with previous findings $[7,32,33]$, heat shock protein (HSP) genes (e.g., grpE, dnaK-J, groES-EL, htpG) and other general stress genes were upregulated under both stresses (Figure 2). The general stress genes are classified into four different categories based on the
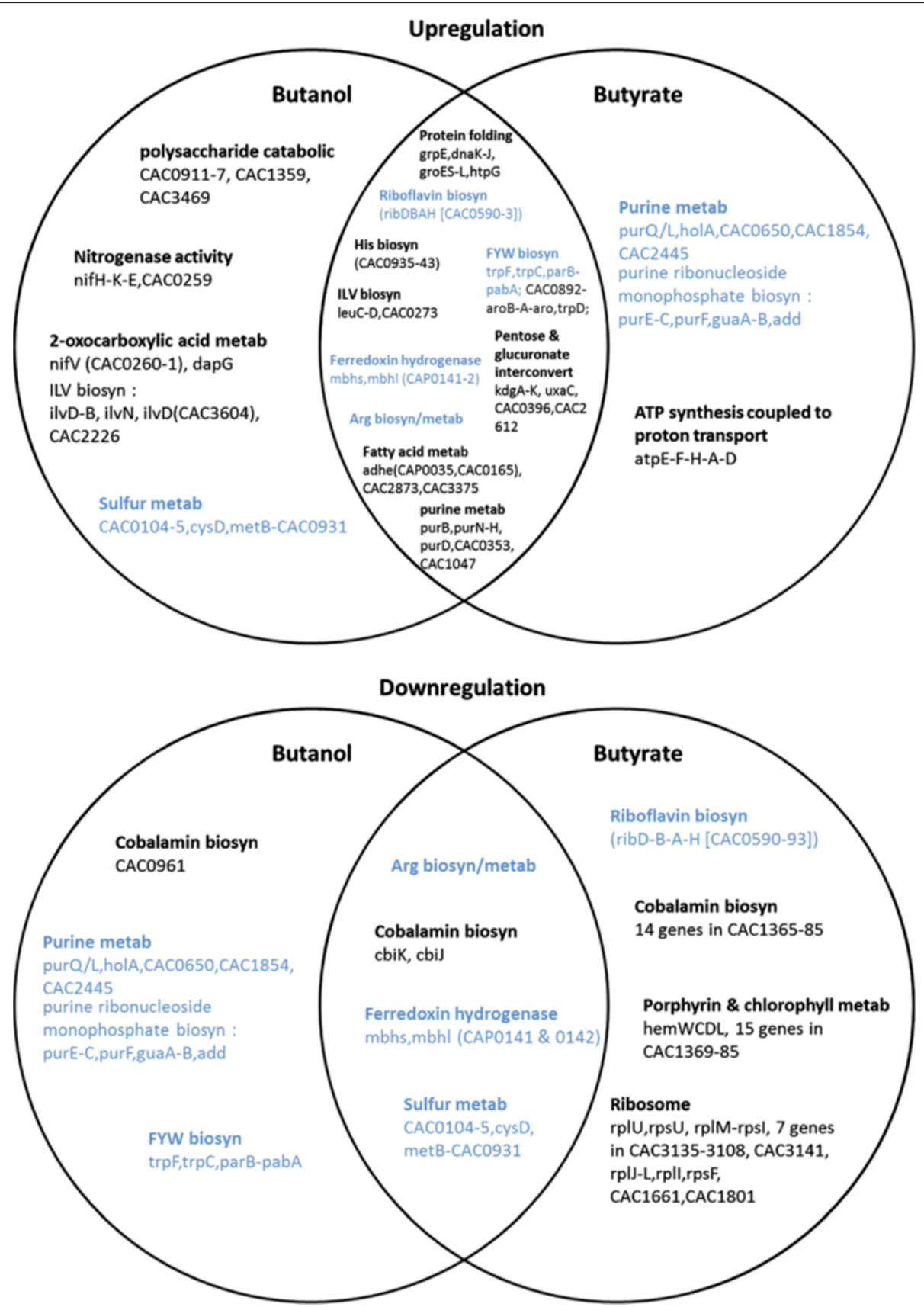

Figure 2 Venn diagrams of selected genes and pathways, which were differentially upregulated or downregulated (with a fold change of $>1.5$ for at least 5 time points per stress experiment) in response to the butanol and butyrate stresses. This list is not all inclusive; a complete list of differentially expressed genes is available in the Additional file 1. The genes and pathways that are both upregulated and downregulated (e.g., some genes are under bimodal regulation at a given stress, and some genes are up-regulated in one stress but downregulated in another stress) are indicated with blue font 
Bacillus subtilis model as Class I, II, III and IV [7,10,34]. Class I stress genes are under the control of HrcA (Figure 3A), and include groES, groEL, grpE, dnaK, dnaJ and $h t p G$. Class III stress genes (Figure $3 \mathrm{~B}$ ) are under the regulation of CtsR (Class Three Stress Protein Regulator) and include $\operatorname{clpP}, \operatorname{clpE}, \operatorname{clp} X$ and the $\operatorname{clp} C$ operon. The operon information used in this study is based on the predicted transcriptional units by Paredes et al. [35]. The HrcA and CtsR regulons are described in detail in the following sections. Class II stress genes in B. subtilis are defined as those under the regulation of the stressspecific sigma factor, $\sigma^{\mathrm{B}}$. As there is no known ortholog for $\sigma^{\mathrm{B}}$ in $C$. acetobutylicum (or any other sequenced Clostridium organism [36]), Class II stress genes are considered absent in C. acetobutylicum. Nevertheless, based on the conservation of the $\sigma^{\mathrm{B}}$ regulated Class II stress proteins and using OMA analysis, we identified 33 proteins in $C$. acetobutylicum that are homologous to the B. subtilis Class II HSPs (Additional file 2: Figure S2). Among them, the only genes that were differentially upregulated (i.e., CAC3187-CAC3192) belong to Class III in C. acetobutylicum, and the rest show weak differential expression, thus further supporting the absence of Class II HSP regulation in C. acetobutylicum. Class IV stress genes are defined as the stress related genes that are not under the control of HrcA, $\sigma^{\mathrm{B}}$ or CtsR. 72 genes that were overexpressed under both butanol and butyrate stress (SAM analysis; fold change $\geq 1.5$ at 0.05 FDR), but that they were not identified as Class I or III genes, were thus identified as Class IV stress genes in C. acetobutylicum (Additional file 2: Figure S3). This is a much larger list than previously identified in this organism $[7,10]$ or any Clostridium. This large Class IV set includes genes from carbohydrate metabolism, histidine

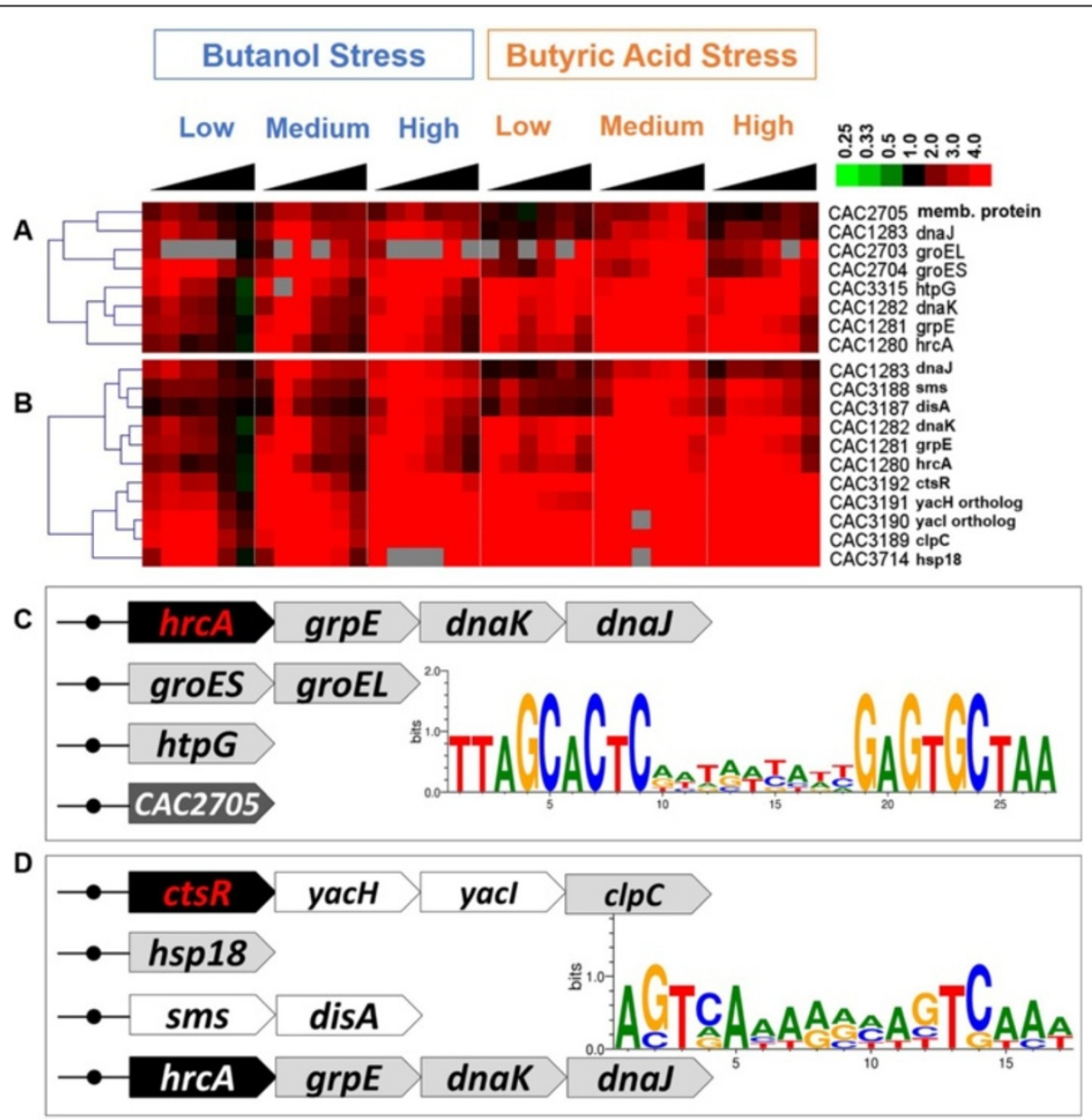

Figure 3 The expression of predicted target genes for HrcA (A) and CtsR (B). The putative regulon structure is shown for HrcA (C) and CtsR (D) (see text for details). Genes in each listed operon are shown by arrows; TF genes are in black, chaperone proteins are in light grey; potential novel genes for a regulon in C. acetobutylicum are in dark grey; presence of TF binding sites are indicated by black circles. The sequence logos (which represent sequence conservation) for HrcA and CtsR binding motifs in C. acetobutylicum are shown in (C) and (D), were created with 6 and 5 binding sites respectively, using WebLogo $[37,38]$. Gene expression data are displayed as in fold change compared to no-stress control cultures at the same timepoint. 
metabolism, the sol-locus genes (CAP0162-CAP0165), genes related to biosynthesis of membrane and cell-wall components, and stress responsive transcriptional regulators/factors (TRs, TFs).

\section{The two HSP regulons: the core of the stress response}

We used a pattern-based approach combined with expression and functional profiling to infer potential TFBSs in the C. acetobutylicum genome, thereby predicting transcriptional regulations and reconstructing stress response network (Figure 1; see Methods for details). Successful identification of TFBSs for many C. acetobutylicum genes suggests good similarity of binding motifs between Clostridium organisms and other $\mathrm{Gram}^{+}$bacteria. Among them, HrcA and CtsR are the two TFs orchestrating the core HSP response.

\section{The HrcA regulon}

Parts of the HrcA and CtsR regulons in C. acetobutylicum have been examined previously. Notably, it was shown that the dnaKJ and groESL operons are HrcA dependent $[10,39]$, whereas $h s p 18$ is not [10]. Our sequence analysis indicates that phylogenetically conserved HrcA binding sites are present upstream of four operons: hrcA-grpEdnaK-dnaJ (CAC1280-CAC1283), groESL (CAC2704CAC2703), CAC2705 and htpG (CAC3315) (Figure 3A \& C), with the first two corroborated by Bahl et al. [39]. All these operons, except for CAC2705, were highly overexpressed during the time course of all the tested butanol and butyrate stresses in this study, which is consistent with previous reports. Although its $\mathrm{HrcA}$ binding site is the same as for its adjacent groESL operon, CAC2705 shows significantly weaker overexpression than the other genes [7], thus suggesting that it is regulated by an additional TF. The function of the membrane protein CAC2705 or any of its orthologs is unknown. Overall, the function of HrcA in $C$. acetobutylicum is likely similar to that in many other well studied organisms, which is to maintain low basal levels of expression of the $d n a K, d n a J$, groESL and $h t p G$ operons in the absence of stress [40]. Upon exposure to stress, these operons are no longer repressed and instead get strongly upregulated. The underlying regulatory mechanism is apparently complex because when $h r c A$ transcription is upregulated, it should lead to the synthesis of more HrcA repressor protein and therefore the repression of these operons should get stronger. But this is in contrast to the fact that these operons get upregulated at the same time as $h r c A$ is upregulated. In B. subtilis, complex post-transcriptional regulations of the $h r c A$ operon, based on mRNA processing and stability, ensures the production of the operon's proteins in the amounts needed by the cell [41]. Additionally, HrcA is activated by the free GroESL protein complex under normal conditions, but during stress, titration of
GroESL by unfolded proteins constrains HrcA activation, thus ensuring additional post-translational control [42].

\section{The Cts $R$ regulon}

Phylogenetic footprinting analysis of the C. acetobutylicum genome revealed the presence of CtsR operator sites upstream of ctsR-yacH-yacI-clpC (CAC3192CAC3189) and hsp18 (CAC3714) [7,10]. By obtaining the $C$. acetobutylicum orthologs for all the target genes of CtsR regulons in RegPrecise, we found additional putative target genes in $C$. acetobutylicum, including the operon of sms-disA (CAC3188-CAC3187) and hrcAgrpE-dnaK-dnaJ (CAC1280-CAC1283) (Figure 3B \& D), whose TFBSs are less similar to CtsR DNA motifs (via Matrix Scan, see Methods section) than those of $c t s R$ and $h s p 18$ operons. Their gene expression patterns align well with $c t s R$ and $h s p 18$ (Figure 3B). The presence of CtsR binding site upstream of $h r c A$ operon confirms the presence of secondary control of the dnaK operon by Homuth et. al. (1999) [41], leading to a cross regulation of CtsR and HrcA, which has been observed in other gram positive organisms [43]. sms (CAC3188) codes for an ortholog for the DNA repair protein RadA in B. subtilis str. 168, which may play a role in the repair of endogenous alkylation damage [44]. DisA (CAC3187) likely participates in a DNA-damage check-point that is active prior to asymmetric division when DNA is damaged, like in B. subtilis [45]. DisA in B. subtilis forms globular foci that rapidly scan along the chromosomes during sporulation, searching for lesions. When a lesion is present, DisA pauses at the lesion site. This triggers a cellular response that culminates in a temporary block in sporulation initiation [45]. CtsR, although a repressor of HSPs, was observed to have higher expression of its transcripts during the onset of stress, similar to HrcA. Repression of CtsR under stress is overcome by its inactivation through phosphorylation of arginine residues by YacI. YacI under normal conditions is bound to $\mathrm{ClpC}$, but is activated under stress by $\mathrm{YacH}$ [46].

\section{Stressor-dependent stress responses Overview}

Many genes in the COG (Clusters of Orthologous Groups) category of amino acid transport and metabolism displayed different patterns of expression under the two stresses. Some of these genes are involved in the biosynthesis of arginine, histidine and tryptophan, and these are discussed in detail below. While some purine metabolism-related genes (purB, purN-H, purD, CAC0353 and CAC1047, Figure 2) were upregulated under both stresses, some of the other genes (purE-C, purF, guaA-B, add, purQ/L, holA, CAC0650, CAC1854 and CAC2445) were upregulated under butyrate stress, but downregulated under butanol stress. 
Other sets of genes that display different expression patterns under the two stresses are genes involved in ATP synthesis genes, cobalamin biosynthesis, and ribosomal protein

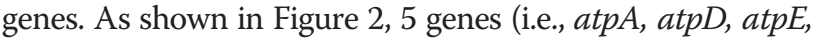
$a t p F$ and $a t p H$ ) (within the CAC2872-CAC2864 operon), involved in ATP synthesis coupled to proton transport, were upregulated under butyrate stress only. Nine genes involved in polysaccharide catabolic processes (CAC0911CAC0917, CAC3469 and CAC1359) were upregulated under butanol stress only, with a few genes among them also upregulated by transient butanol pulse in chemostat cells [32]. The majority of the genes involved in cobalamin biosynthesis were down-regulated under butyrate stress but not under butanol stress, which is consistent with a previous study [47]. They include 14 genes, cbiM, cbiG, cobT, cbiP, cobB, cbiC, cbiD, cbiT, cobI/cbiL, cbiF/cobM, cbiH/ cobJ, cobU, cobS and $c o b C$, which are part of the CAC1365CAC1386 operon. This suggests a lowered need for cobalamin for the strain under butyrate stress. Cobalamin is a necessary cofactor for various reactions involving rearrangements including glycerol dehydratases for glycerol metabolism and transmethylation for the formation of methionine from homocysteine [47]. 27 out of 48 ribosomal protein-encoding genes were downregulated under butyrate stress. Among them, 18 were only downregulated under butyrate stress but not so under butanol stress (Figure 2 and Additional file 2: Figure S4). These data suggest that translation is suppressed during butyrate stress at all three stressor levels (low, medium and high), consistent with the previous studies [7].

\section{Amino- and nucleic-acid biosynthesis}

Arginine biosynthesis related genes (e.g., argF/I (CAC 0316), argG-argH (CAC0973-CAC0974), argB-argD (CAC2389-CAC2388), argC-argJ (CAC2390-CAC2391), carB (CAC2644), carA (CAC2645) and CAC3619 (coding for amino acid $\mathrm{ABC}$ transporter component)) exhibited dose- and time-dependent expression for both butanol and butyrate stresses (Figure 4A and Additional file 2: Figure S6). For example, their expression was more strongly upregulated at the low compared to high butyrate stress. In addition, they show different expression patterns from those reported by Alsaker et al. in 2010 [7] when comparing the low and medium levels of butyrate stress at 30,45 , and 60 min post stress. These differences can be ascribed to the different experimental conditions used in the two studies. In the 2010 study, Clostridial Growth Medium (CGM) containing yeast extract was used without $\mathrm{pH}$ control in static-flask cultures, whereas in the current study, a defined medium was employed in agitated bioreactors with $\mathrm{pH}$ controlled at 5.0. Based on the fact that butyrate inhibition is $\mathrm{pH}$ dependent [7], and that $\mathrm{pH}$ dependent growth play a crucial role in the cellular adaptation [48], our assessment is that these difference are predominantly due to the impact of $\mathrm{pH}$, but also perhaps the presence of arginine or intermediates of its biosynthesis in the complex CGM medium. In Escherichia coli, and several other organisms, the arginine decarboxylase/ antiporter-dependent acid-resistance (AR) system 3 (AR3) [49], is one of the four known AR systems protecting cells from acid stress. But as already discussed [50], there are no ortholog genes to these or the other AR systems in $C$. acetobutylicum. Nevertheless arginine biosynthesis and transport in combination with culture $\mathrm{pH}$ apparently play an important role in carboxylic acid stress.

Nine genes involved in histidine biosynthesis (hisZ-hisGhisD-hisB-hisH-hisA-hisF-hisI-hisE (CAC0935-CAC0943) displayed complex expression patterns post stress (Figure 4B). Interestingly, induction of genes for histidine biosynthesis has recently been shown to contribute to acid tolerance in Lactobacillus casei [51]. In addition, a histidine decarboxylation pathway introduced in Lactococcus lactis improved survival to acid stress [52].

Similarly, the tryptophan biosynthesis genes in the CAC3163-CAC3157 operon (parB, pabA, trpD, trpC, $\operatorname{trp} F, \operatorname{trp} B$ and $\operatorname{trp} A)$ show strong dose dependence on butanol concentration (Additional file 2: Figure S6), where low butanol stress leads to strong overexpression at the early timepoints, but mostly to downregulation for medium and high butanol stress. Under ethanol stress, tryptophan genes were reported to be upregulated and functionally tested in Saccharomyces cerevisiae [53]. For butyrate stress, there was upregulation for all 3 levels of stress, which is unlike the Alsaker et al. 2010 study, where a weak downregulation was found under butyrate stress [7]. As discussed, these differences may be related to the presence of yeast extract and the lack of $\mathrm{pH}$ control in the 2010 study. Cysteine metabolism genes also show a butyrate dose-dependent expression (Figure 4C \& Additional file 2: Figure S6).

The branched-chain amino acids (BCAAs, i.e., valine, leucine and isoleucine) are involved in the synthesis of branched chain fatty acids through the formation of $\alpha$ keto acids. It has been suggested [7] that BCAAs are incorporated into membrane components to generate more rigid membrane aiming to counteract the solvation effect of toxicity solvents. Homeoviscous membrane changes involving modifications of membrane fatty acid composition to counteract the fluidizing effects of butanol and other solvents have been extensively discussed in the literature $[15,16]$. In Streptococcus murants, the BCAA aminotransferase encoded by $i l v E$ was shown to protect against acid stress [54]. Thus, BCAAs may serve in multiple roles in dealing with both solvent and acid toxicity. The genes ilvC (CAC0091) and leuC-leuD-leuB$i l v D$-ilvB (CAC3173-CAC3169) are involved in valine, leucine and isoleucine biosynthesis. Under butanol stress (Additional file 2: Figure S6), expression of these 


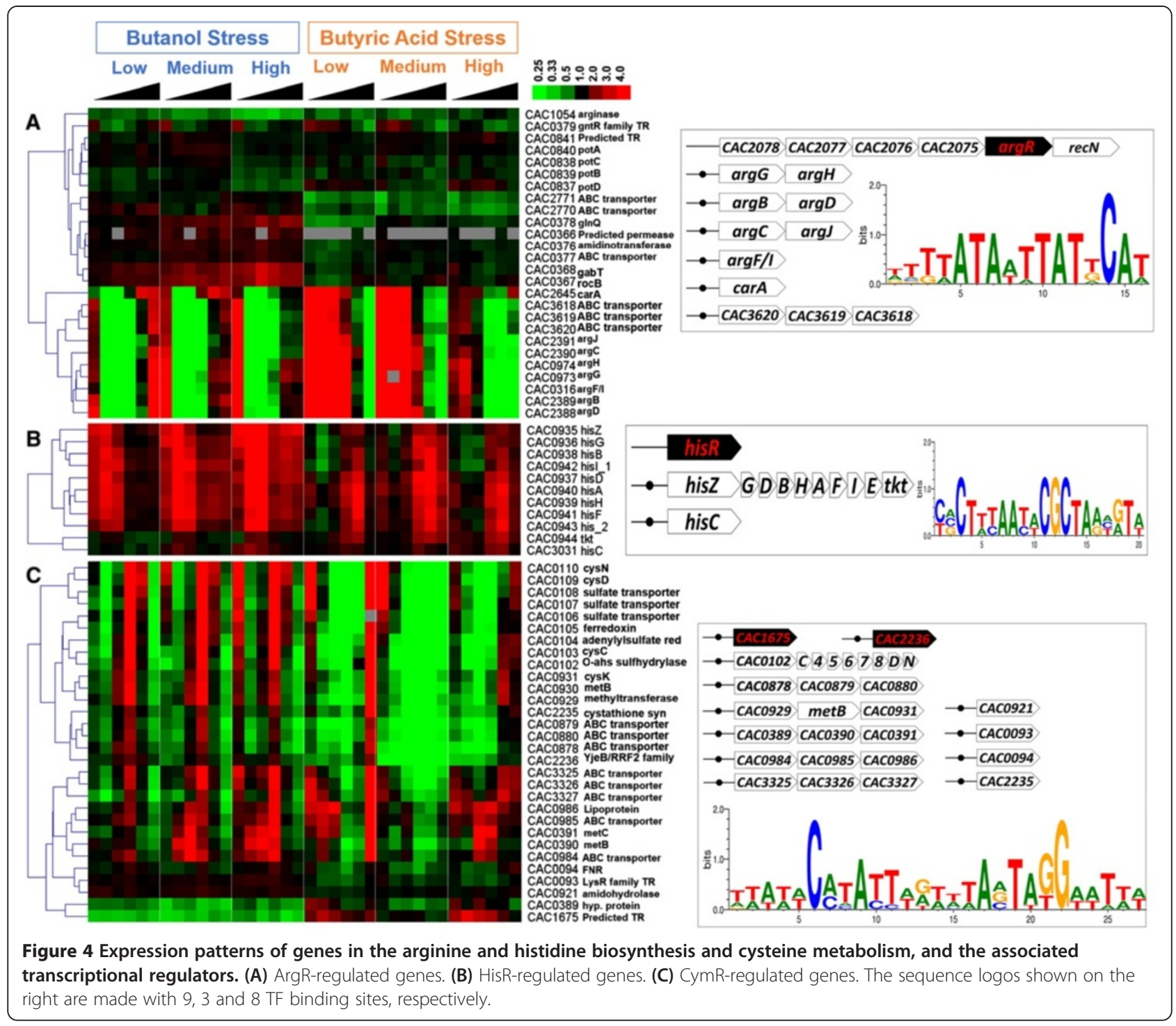

BCAA genes is dose dependent displaying stronger upregulation at high butanol levels compared to low or medium levels. These genes display a more complex pattern under butyrate stress, with significant downregulation under high stress.

Several genes involved in purine metabolism (Additional file 2: Figure S5 and S6), including purE- purC (CAC1390CAC1391), purF-purM-purN-purH (CAC1392-CAC1395), purD (CAC1396) and purQ/purL (CAC1655), displayed distinct expression patterns for different butanol stress levels: at low and medium levels of butanol, these genes were weakly upregulated while at high level of butanol, they were downregulated. The response of these genes to butyrate stresses was strong in this study, and displayed different kinetics and strengths compared to results from Alsaker et al. [7]. Here, these genes show the strongest upregulation at high butyrate stress level, which may suggest their involvement in acid resistance. Insertional mutagenesis of the Lactococcus lactis purine metabolism gene guaA led to increased acid resistance [55]. Purine metabolism also shares a large number of genes and pathway intermediates with histidine metabolism, which, as already discussed, were also found to be differentially expressed under stress. It is noteworthy that metabolic pools (and, notably, purine nucleotides) impact acid tolerance [55].

\section{Differential expression of transcriptional regulators: overview}

Given that the two stressors elicit several common but also many distinct responses per our discussion above, we aimed next to identify the TFs that might orchestrate these responses. The expression profile for the 164 significantly differentially expressed TRs in C. acetobutylicum is shown in Additional file 2: Figure S7. K-means 
clustering of these 164 TRs identified common and distinct expression patterns for TRs under the two metabolite stresses. Clusters containing TRs that are upregulated (Additional file 2: Figure S7 A, F \& I) and downregulated (Additional file 2: Figure S7 C, D \& E) at the same time under both stresses can be linked to general stress response, while clusters consisting of TRs with distinct expression patterns for each stress (Additional file 2: Figure S7 B, G, H \& J) are involved in orchestrating the stressor-specific response.

Because a large fraction of the aforementioned 164 TRs remain non-annotated (Additional file 3), we used phylogenetic footprinting and comparison with RegPrecise DNA motifs (Figure 1), and thus many TFBSs were successfully identified in C. acetobutylicum, and are shown in various figures. Many such identified TFBSs correspond to a subset of the core TFs in Bacillales. These include regulators that control the metabolism of amino acids and nitrogen (ArgR, CodY and CymR), carbohydrates (CcpA and CggR), biotin cofactor (BirA), fatty acids (FapR) and nucleotides (NrdR and PurR). These regulators also include TFs for metal homeostasis (Fur, MntR and Zur), respiration (Rex), sporulation (Spo0A), stress responses (CsoR, CtsR, HrcA, LexA and PerR), as well as the chromosomal replication initiation regulator DnaA [56]. Next, we focus on selected regulators with target genes significantly differentially expressed, per our discussion above, in butanol and/or butyrate stressed C. acetobutylicum. We start with the three stress-related regulators, which, unlike CtsR and HrcA, have not yet been examined at the systems level in Clostridium. We then discuss transcriptional regulators of amino acid and purine metabolism that appear to be part of the specialized metabolite stress response.

\section{The core stress-associated transcription factors engaged in the butanol and butyrate stress response LexA}

LexA is well-known as the primary TF controlling the SOS response, which is an inducible DNA repair system that allows bacteria to survive sudden increases in DNA damage [57]. Even though it has been well studied in many bacteria, the LexA regulon has not been examined in $C$. acetobutylicum so far. Therefore it was important to examine which genes might be under LexA control in C. acetobutylicum and whether these SOS response genes are differentially regulated under butanol or butyrate stress. Butyrate stress, in particular, may cause acidinduced DNA damage, which is frequently compared to oxidative stress [58]. RSAT footprint analysis led to the identification of four genes/operons with highly conserved cis-elements in their promoter regions that include LexA binding sites known as SOS boxes [59]. These are lexA (CAC1832), recA (CAC1815), uvrBuvrA-CAC0504-CAC0505-CAC0506-CAC0507-uvrC-
CAC0509-murB (CAC0502-CAC0510), and CAC3343CAC3344. In addition, the orthologs in C. acetobutylicum for all target genes in LexA regulons from RegPrecise were collected using OMA search, and scanned for LexA binding site in their operon leader sequences. The operons with an identifiable LexA binding site (p-value $<1 \mathrm{e}-3$ ) were collected and the microarray data for their gene members are shown in Figure 5. Hierarchical clustering shows that $\operatorname{lex} A, \operatorname{rec} A$ and the $u v r B$ operon are clustered well. This is consistent with the possibility that they are functionally correlated, like in other well studied bacteria $[60,61]$. Moreover, even though no ortholog of CAC3343 was listed as under LexA control in RegPrecise, our footprinting analysis together with expression data suggests that the CAC3343-CAC3344 operon is also putatively controlled by LexA. CAC3343 encodes a putative DNA modification/repair radical SAM protein and CAC3344 encodes a protein of unknown function. In addition, the operon of $s b c D-s b c C$ (CAC2737-CAC2736; sbcD: DNA repair exonuclease; $s b c C$ : ATPase involved in DNA repair) is clustered closely to $r e c A$, and its promoter region shows a potential LexA binding site (p-value $<1 e-3)$. The UvrABC repair system catalyzes the recognition and processing of DNA lesions. A damage recognition complex composed of 2 UvrA and 2 UvrB subunits scans DNA for abnormalities. Regulation of the UvrABC system by LexA is well established in many bacteria, including $E$. coli and B. subtilis $[60,61]$, but has not been examined in Clostridium. The more significant upregulation of $u v r B$ under butyrate stress compared to butanol stress suggests that DNA damage may be more prevalent under butyrate stress. As discussed, butyrate stress affects the cells similar to oxidative stress by damaging DNA, proteins and lipids [58]. Therefore, we conclude that the LexA regulon in C. acetobutylicum contains at least the following genes/operons (Figure 5): lexA (CAC1832), recA (CAC1815), uvrB-uvrA-CAC0504-CAC0505-CAC0506CAC0507-uvrC-CAC0509-murB (CAC0502-CAC0510), CA C3343-CAC3344, and $s b c D-s b c C$ (CAC2737-CAC2736).

\section{Rex: the redox sensor}

Rex (encoded by CAC2713) is a member for the Rex family, which includes regulators that modulate transcription in response to changes in cellular NADH/NAD + levels and more generally the redox state [62]. It was previously demonstrated that Rex is a redox-sensing transcriptional repressor that regulates solventogenesis in C. acetobutylicum [63]. In our microarray experiments, rex was downregulated under butyrate stress. Our footprinting analysis identified 6 operons with potential Rex binding sites in their promoter regions: hydA (CAC0028), nirC-asrA-asrB-asrC (CAC1512CAC1515), crt-bcd-etfB-etfA (CAC2712-CAC2709), CA C2713, CAC2873, and adhE2 (CAP0035), the fusion- 


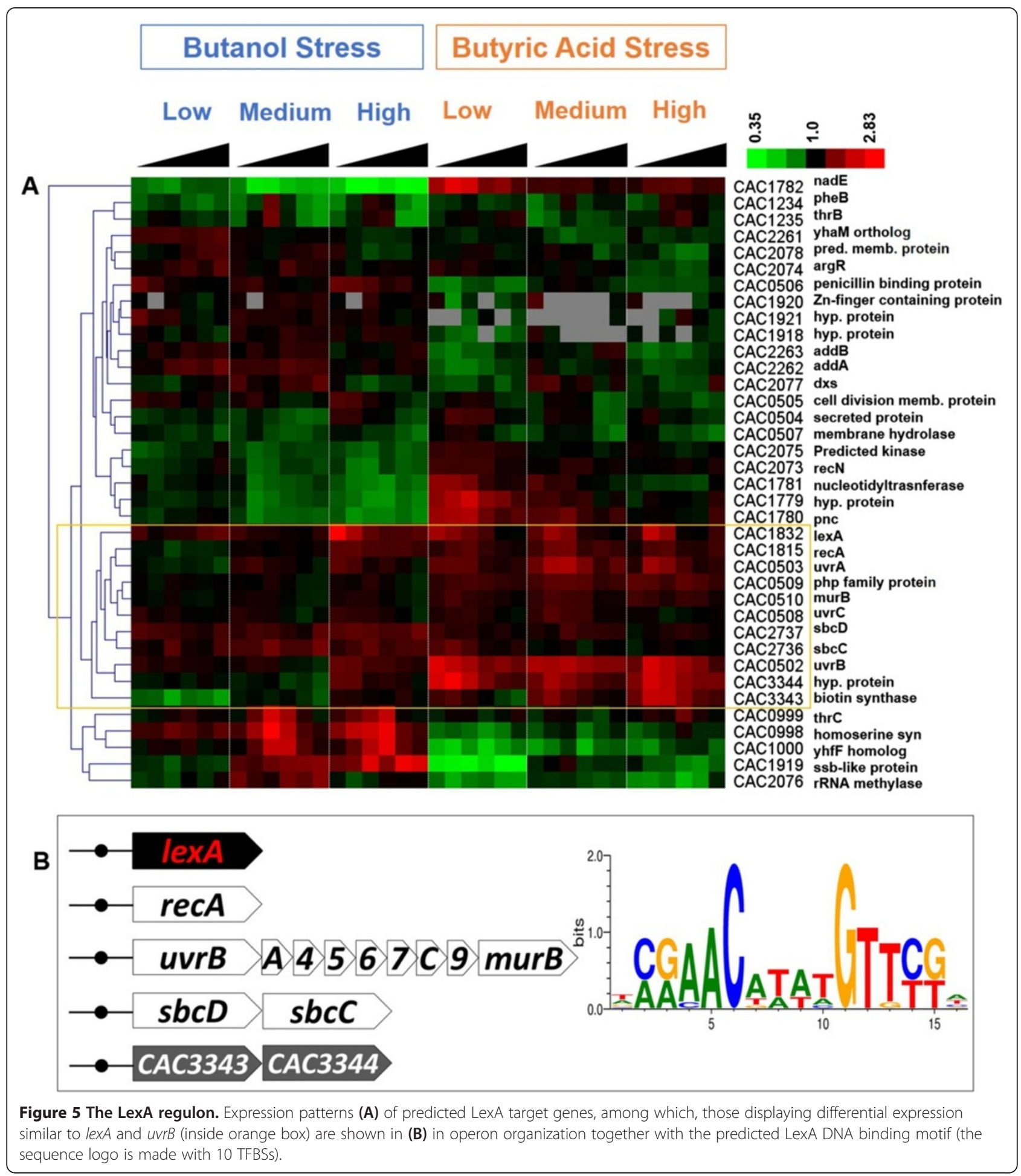

protein aldehyde/alcohol dehydrogenase, which is highly homologous to adhE1 (CAP0162). In addition, based on orthology to target genes in Rex regulons from RegPrecise and identification of putative Rex binding sites (Matrix Scan with the Rex-binding DNA motif in Clostridiales from RegPrecise, p-value $<1 \mathrm{e}-3$ ), the following operons were found to be putative targets for Rex regulation in C. acetobutylicum as well: CAC0014-serA (CAC0014-CAC0015), ldh (CAC0267), gapC (CAC0709), CAC0827, nadAnadB-nadC (CAC1025-CAC1023), CAC2229, CAC2872atpB-atpE-atpF-atpH-atpA-atpG-atpD-atpC （CAC2872CAC2864), adhE1-ctfA-ctfB (CAP0162-CAP0164). The 
expression patterns for the genes in these operons are shown in Figure 6A. It is noteworthy that $a d h \mathrm{E} 2$ is highly upregulated, together with adhE1-ctfa-ctfb. The latter is the set of genes responsible for butanol production in this organism [5]. As discussed above, these genes are also part of Class IV stress response genes.

\section{PerR}

PerR (encoded by CAC2634) represses the expression of most of the genes involved in oxidative stress response, such as $\operatorname{rbr} 3 A-r b r 3 B, d f x, r d$, nror, fprA1 and fprA2, which code for reverse rubrerythrins, desulfoferrodoxin, rubredoxin, NADH-rubredoxin oxidoreductase (NROR), and oxygen-reducing flavoproteins, respectively $[64,65]$. PerR probably acts as a peroxide sensor [65]. By facilitating reactive oxygen species
(ROS) scavenging, PerR plays an important role in the oxidative stress defense system in C. acetobutylicum, which is an obligate anaerobe. Cells lacking this gene exhibit enhanced aerotolerance and increased $\mathrm{H}_{2} \mathrm{O}_{2}$ resistance. Deletion of per $R$ does not affect the intracellular level of iron but increases two-fold that of zinc [64]. Several direct targets of PerR were proposed previously by Hillmann et al., through performing a genome-wide search for potential PerR binding sites using Virtual Footprint software in combination with global transcription analysis of the perR deletion mutant against the wild type [65]. Our microarray data for these genes are shown clustered in Figure 6B. Apparently, the Alsaker et al. (2010) study showed strong upregulation in these genes for the butyrate stress, but this was not the case in our new study.

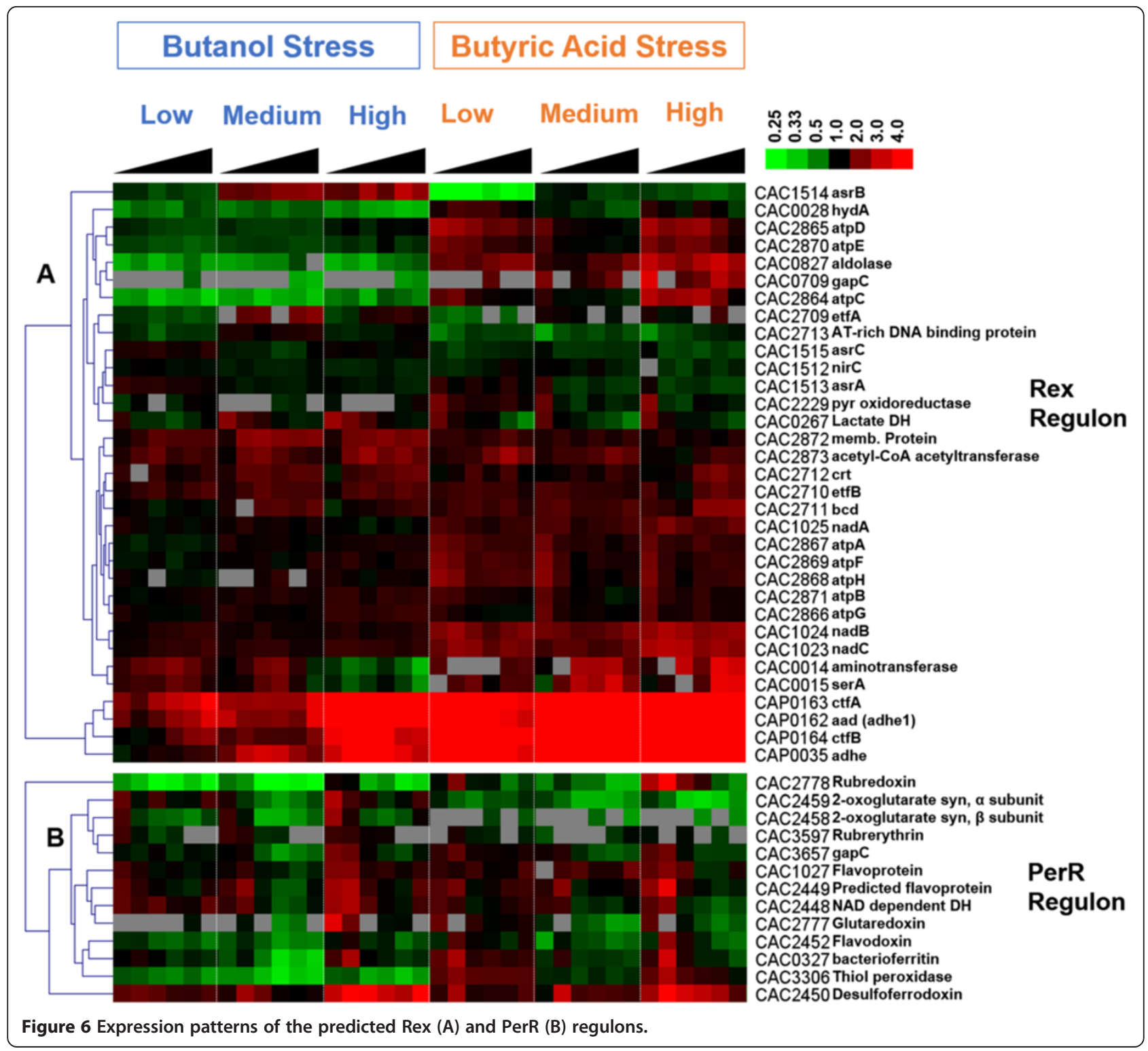


This may suggest that our new fermentative and culture conditions (with $\mathrm{pH}$ control) lead to less oxidative stress and therefore these PerR-regulated genes remained largely repressed or only mildly derepressed.

\section{Amino- and nucleic-acid transcriptional regulators en- gaged in the metabolite stress responses ArgR: the arginine repressor}

The arginine repressor (ArgR) is known as a master regulator of arginine biosynthesis and catabolism in bacteria in response to intracellular arginine levels [66]. As several genes related to arginine biosynthetic process show strong bimodal expression under both butanol and butyrate stress (Additional file 2: Figure S6), it is of interest to explore the potential involvement of ArgR. ArgR in C. acetobutylicum is encoded by CAC2074, the $5^{\text {th }}$ gene in the CAC2078CAC2073 operon. The ArgR in E.coli is known to autoregulate its own transcription [67]. However, it does not appear to be autoregulated in C. acetobutylicum, because the promoter region of its operon does not have an identifiable ArgR binding motif (shown in Figure 4A), based on Matrix scan (p-value $<1 e-3$ ). Three operons with genes in arginine biosynthetic activity have conserved cis-elements in their promoter regions that align well with the known ArgR motifs from RegPrecise (Figure 4A). These operons are $\operatorname{argG-\operatorname {argH}}$ (CAC0973-CAC0974; $\operatorname{argG}$, argininosuccinate synthase; $\operatorname{argH}$, argininosuccinate lyase), $\operatorname{argB}$-argD (CAC2389-CAC2388; $\operatorname{argD}, \mathrm{N}$-acetylornithine aminotransferase; $\operatorname{argB}$, acetylglutamate kinase), and $\operatorname{argC}$-argJ (CAC2390-CAC2391; argC, N-acetyl-gamma-glutamylphosphate reductase; argJ, ornithine acetyltransferase). Searching for orthologs of the target genes in ArgR regulogs from RegPrecise (a regulog is defined as a set of coregulated genes for which the regulatory sequence has been conserved across multiple organisms [22]) and scanning with the ArgR DNA-binding motif in Clostridiales, several additional operons were identified as potentially also regulated by ArgR. Among them, argF/I (CAC0316), carA (CAC2645) and CAC3620-CAC3618 (amino acid ABC transporter components) show a more similar expression pattern to those of $\operatorname{argGH}, \operatorname{argBD}$ and $\operatorname{argCJ}$ (Figure 4A) than the other genes. Interestingly, this finding suggests that CAC3620-CAC3618 encode a putative arginine transport system, potentially controlling arginine uptake from the environment, as proposed previously for an arginineresponsive gene regulation [68]. Altogether, the ArgR regulon in C. acetobutylicum likely includes at least 6 operons/ genes (Figure 4A). Furthermore, our de novo motif prediction with MOTIFATOR [69] suggests an ArgR binding site upstream of CAC0380. CAC0380 was previously annotated as a periplasmic amino acid-binding protein, but now with our prediction of ArgR binding site for this gene, we suspect it is an arginine-binding protein with a possible role in AR3 mechanism.

\section{HisR: histidine biosynthesis regulator}

As discussed, histidine biosynthesis genes are dynamically upregulated under both butanol and butyrate stress (Additional file 2: Figure S6). RSAT phylogenetic footprinting [20] analysis shows a well conserved motif upstream of the his $Z$ genes in organisms of the Clostridia class. The C. acetobutylicum his $Z$ gene is in an operon of 10 genes (CAC0935-CAC0944), including hisZ, hisG, hisD, hisB, hisH, hisA, hisF, hisI, hisE and tkt. Nine of them show a dynamic upregulation under both butanol and butyrate stress (Figure 4B). The cis-elements in the upstream region of his $Z$ genes in Clostridia class (identified by phylogenetic footprinting) bear high similarity to the DNA motif recognized by HisR of Staphylococcaceae [22] (Additional file 2: Figure S9). According to OMA [26,70], CAC2675 is an ortholog of hisR, which is predicted to be present in several other $\mathrm{Gram}^{+}$organisms in RegPrecise. Based on its orthology to the corresponding target genes assigned in HisR regulogs in RegPrecise and identification of potential HisRbinding site in its promoter region, hisC (CAC3031) is also a putative target gene for HisR. To sum, the HisR regulon in C. acetobutylicum is proposed to include the hisZ-tkt operon and hisC (CAC3031) (Figure 4B).

\section{CymR: cysteine metabolism repressor}

In B. subtilis, CymR is a master repressor of cysteine metabolism. It controls the expression of genes involved either in cysteine synthesis from sulfide (cysK), sulfonates (ssu), or methionine $(m c c A B)$ or in cysteine uptake $(t c y P)$ [71]. The activity of CymR is positively regulated by CysK in response to cysteine availability [72]. When cysteine is present, the pool of O-acetylserine (OAS) is low, which leads to the formation of a CymR-CysK complex and transcriptional repression of the CymR regulon occurs. In the absence of cysteine, the OAS pool is high and the CymR-CysK complex is mostly dissociated, leading to a faster dissociation of CymR from its DNA targets and the lifting of CymRdependent repression [71,72]. Proteins CAC2236 and CAC1675 are the best BLAST hits for the B. subtilis CymR [71] in C. acetobutylicum. Both proteins belong to the Rrf2 family, with winged helix-turn-helix transcription repressor DNA-binding domain. RegPrecise database assigns CAC1675 as iron-sulfur cluster assembly transcription factor IscR. Indeed, CAC2236 shows slightly higher sequence similarity to CymR than CAC1675 (64.63\% vs. 60.69\%), whereas CAC1675 has better sequence similarity to IscR in E. coli than CAC2236 does (53.09\% vs. 49.39\%). Therefore it is possible that CAC2236 functions as CymR in C. acetobutylicum, and CAC1675 as IscR. In C. acetobutylicum, CAC2235, immediately downstream of CAC2236, codes for a cysteine synthase (CysK). In addition, the STRING network for CAC2236 further supports its involvement in regulating cysteine metabolism [73]. On the other hand, RSAT footprinting analysis indicates that CAC1675 has a 
promoter element conserved in Clostridia class that partially aligns well with the CymR motif from Staphylococcaceae (Figure 4C). In fact, the IscR- and CymR-binding DNA motifs share quite significant sequence similarities (Figure 4C) [22]. Furthermore, both CAC2236 and CAC1675 have identifiable CymR-binding sites when searched with the PWM built from TFBSs in Staphylococcaceae [22].

Seven operons with potential CymR-binding sites in their promoter regions were identified based on orthology to the CymR target genes in Bacillales and Staphylococcaceae in RegPrecise, and with pattern matched in promoter regions using Matrix Scan (p-value $<1 \mathrm{e}-3)$. They include operons CAC0102CAC0110 (including genes such as cysC (CAC0103), cysD (CAC0109), cysN (CAC0110); protein CysC and CysN are involved in hydrogen sulfide biosynthetic process) and CAC0878-0880 (amino acid ABC transporter system) (Figure $4 \mathrm{C}$ ). Making use of the distinct expression pattern of CAC0102-0110 and CAC0878-0880, the other cysteine-metabolism related genes/operons with similar expression pattern, i.e., cysK (CAC2235), operons of CAC0929-0931 and CAC3325-3327, were further analyzed for CymR-binding sites. Note that cysK and CAC0931 are putatively involved in cysteine biosynthesis from serine and in cysteine synthase activity respectively, whereas CAC3325-CAC3327 encodes protein orthologous to Lcystine-binding protein TcyABC transporter in Staphylococcus carnosus. Indeed, cysK (CAC2235), CAC0929 and CAC3325 have good match to the CymR-binding motif in their promoter regions (for the latter two, the predicted CymR-binding site is located more than 300 nts upstream their translation start codons). We conclude that the CymR regulon in $C$. acetobutylicum likely includes at least the genes shown in Figure 4C.

\section{PurR: repressor of purine metabolism}

PurR (encoded by CAC3224) is a member of PurR family, which serves as repressor of purine metabolism [7]. CAC3224 is upregulated under butanol stress, but not so under butyrate stress, which may explain the upregulation of several purine metabolism related genes when under butyrate stress. Interestingly, the majority of $C$. acetobutylicum genes orthologous to the PurR target genes in RegPrecise seem to contain only half of the PurR-binding DNA motif in Bacillales, which is a palindromic sequence comprised of two inverted repeats.

Complexity of the metabolite stress response as captured by the STRING-based stress response network (SRN)

The high-confidence functional interactions predicted by STRING (Confidence Score $\geq 0.700$ ) were used as edges to construct the butanol/butyrate stress response network (SRN), which is shown in Additional file 2:
Figure S8. Cross-interactions are prevalent between most of the key regulons discussed above. Since the majority of the transcription factors and their target genes are highly conserved in a variety of organisms of the Clostridium genus (Additional file 4: Table S4), this SRN is likely largely applicable to the other Clostridium organisms, In addition, the complexity observed in these two core metabolite stresses may require additional layers of regulation, such as differential mRNA degradation and the regulation at transcriptional and post-transcriptional levels by non-coding small RNAs (sRNAs). The role of sRNA under metabolite stress has been investigated for butanol and butyrate stress based on RNAseq data, and quite a few stress-responsive sRNAs have been identified (Venkataramanan et al. 2013, BMC Genomics) [74]. Several sRNAs were particularly up-regulated under both the metabolite stresses. They include 6S RNA (regulating availability of specific sigma factor and hence the expression of genes under their regulation), tmRNA (transfermessenger RNA, which recycles ribosomes and ensures their availability during a change in the transcriptional and translational machinery of the cells along with labeling aberrant protein for degradation), SRP (signal recognition particle RNA, which regulates the trans-translation of the membrane bound proteins), solB (the repressor of the sol operon) and SAM riboswitch (regulating the expression of sulfur amino acid metabolism). Identifiable or likely targets of these sRNAs would suggest that these and other sRNAs are likely involved in regulating at least some of the genes in the core regulons discussed in this study.

\section{Conclusions}

Gene regulatory networks play essential roles in living organisms to respond to both external environmental and internal metabolism changes. Understanding the connections and the activity levels of regulators is indispensible for the gene regulatory network research. This study produced a large set of high-resolution temporal transcriptional data. Using phylogenetic footprinting analysis and orthology inference, combined with pattern-based TFBS detection, the regulatory interactions proposed from our study provide important connections between transcription factors and their target genes that are important for butanol and butyrate stress response in C. acetobutylicum. Utilizing predicted transcription factor activities in combination with transcriptome data [75] can be a future direction for reconstructing gene regulatory network of $C$. acetobutylicum. It is known that the analysis of transcriptome data does not always permit identifying the primary cause of a phenomenon observed. There are various levels of regulations apart from transcriptional ones, e.g., epigenetic regulation, translational regulations, mRNA stabilities, post-translational modifications. It remains a grand challenge to integrate all these 
regulation layers together to reconstruct a holistic stress response network. But promising systems biology methods are in development to provide solutions to the problem.

\section{Methods \\ Microarray experiments and data analyses Strain and growth conditions}

C. acetobutylicum ATCC 824 was grown in a defined clostridial growth medium (40 g/L glucose) in BioFlo 310 Fermentors (New Brunswick Scientific, Edison, $\mathrm{NJ}$ ) equipped with controllers for $\mathrm{pH}$, temperature, and agitation [12] to an $\mathrm{OD}_{600}$ of 1.0 and stressed with appropriate concentrations of butanol (low - $30 \mathrm{mM}$, med - $60 \mathrm{mM}$, high - $90 \mathrm{mM}$ ), or butyrate (low - $30 \mathrm{mM}$, med - $40 \mathrm{mM}$, high - $48 \mathrm{mM})$. Samples were taken at 6 time points post-stress $(0 \mathrm{~min}, 15 \mathrm{~min}, 30 \mathrm{~min}, 45 \mathrm{~min}$, $60 \mathrm{~min}$ and $75 \mathrm{~min}$ ) for RNA isolation. Samples from non-stressed cultures (i.e., $0 \mathrm{mM}$ butanol) were also collected at the same 6 time points, for control. Experiments were carried out in triplicates (2 replicates for preparing labeled-cDNA for microarray hybridization and the third replicate for q-RT-PCR using unlabeled cDNA, for validating the microarray results). All experiments were run in replicates of 4 and the most similar 3 were selected for RNA isolation. The growth curve and product formation patterns are summarized in Additional file 2: Figure S9.

\section{RNA isolation and labeled cDNA generation}

Samples for RNA isolation were collected by centrifuging $15 \mathrm{~mL}$ of cultures at $5,000 \mathrm{rpm}$ for $10 \mathrm{~min}$ and the cell pellets were frozen at $-80^{\circ} \mathrm{C}$. Before extracting RNA, the pellets were thawn and RNA was extracted using Qiagen's RNeasy Mini Kit as described earlier [5]. cDNA generation and its subsequent amino allyl labeling were performed as described [47].

\section{Microarray analysis}

Microarray analysis was performed using Agilent custom arrays $(4 \times 44 \mathrm{~K}$ arrays, GEO accession number GPL10908) by hybridizing $250 \mathrm{ng}$ of $\mathrm{Cy} 3 / \mathrm{Cy} 5$ labeled cDNA hybridized against $250 \mathrm{ng}$ oppositely labeled (Cy5/Cy3) common reference pool (containing equal amount of $2.5 \mu \mathrm{g}$ labeled cDNA from each of the 48 samples) at $65^{\circ} \mathrm{C}$ for $16-18$ hours. Separate reference pools were created for butanol and butyrate stress, respectively. Following hybridization, the slides were washed and scanned in an Agilent scanner; image analysis was carried out using Agilent's Feature Extraction Software (v9.5.1). Normalization was carried out using the LOESS method in $\mathrm{R}$ (Limma package from Bioconductor [76]) using a custom script/algorithm developed in Papoutsakis lab based on SNNLERN (Segmental Nearest Neighbor normalization method) [77]. Normalized outputs contained averaged, normalized values of replicates and dye-swaps with respect to the common reference pool. The microarray data can be accessed at GEO through the access numbers GSE48031 and GSE48039, respectively.

The data were analyzed by pairwise and point-by-point comparison to the non-stress control using significant analysis of microarrays (SAM) [27] in TIGR MeV suite 4.8.1 [78]. For a gene to be identified as being differentially expressed for a metabolite stress, it had to be significant (5\% FDR for SAM analysis) and have at least five time points out of the 18 with expression changes (6 time points for each stress level of Low, Medium and High) over 1.5 fold (up or down) for that metabolite stress. To be considered as bimodally expressed, the gene had to have at least five time points for 1.5 fold up and five time points for 1.5 fold down individually for that metabolite stress. Normalized ratios were grouped by K-means clustering (TIGR MeV, version 4.8.1 [78]) and visualized with heat (or Eisen) plots [79] using TIGR MeV [78]. The K-means clustering was carried out with Euclidean distance metric. We chose K-Means clustering because the number of clusters produced can be directly controlled by the user-defined parameter $\mathrm{K}$ without having to somewhat arbitrarily cut a clustering tree like for the case of hierarchical clustering. The microarray data was validated using q-RT-PCR for 6 genes, which were validated for upregulation (CAP0102, CAC1391, CAC1405 \& CAC3190), downregulation (CAP0102, CAC0766 \& CAC1806), butanol stress (CAP0102, CAC1405, CAC1806 \& CAC3190) and butyrate stress (CAP0102, CAC1391 \& CAC0766). CAC3571 was used as the house keeping gene. The comparison between Q-RTPCR and microarrays are summarized in Additional file 2: Figure S10.

\section{Transcription factor binding site analysis}

DNA sequences up to $300 \mathrm{nt}$ upstream from the start codon of genes in C. acetobutylicum were obtained using RSAT Sequence tools. Given a user-provided query gene, RSAT footprint-discovery analysis provides PWMs for over-represented oligonucleotides (words) or spaced pairs thereof (dyads) for the query gene's orthologs in a user-defined taxon [20]. The obtained PWMs were compared against the PWMs of known TF binding DNA motifs from three resources: RegPrecise [22], RegTransbase [23] and PRODORIC databases [24], with the tool Tomtom in suite MEME [25]. The results, together with OMA ortholog search in C. acetobutylicum genome for the predicted TFs and target genes (TGs) defined in RegPrecise [22,26], allowed the inference of TFs and their target genes in $C$. acetobutylicum. RSAT Matrix Scan is used to scan the C. acetobutylicum upstream sequences with PWMs to identify instances of the corresponding motifs (putative TFBSs). 


\section{Identification of genes associated with stress responses from the literature and databases}

We collected 318 B. subtilis stress related proteins through data mining and text mining. With BLAST search between B. subtilis and C. acetobutylicum, 515 C. acetobutylicum proteins matching to 209 out of those 318 B. subtilis stress proteins are obtained. We also obtained PPIs from STRING database, a pre-computed database for the exploration of protein-protein interactions (PPIs). The 9.05 version of STRING, which was the newest version at the time of the study used here, covers approximately 5 million proteins from 1133 different organisms [73]. We integrated "Actions" data [which include actions such as inhibition, activation, reaction, catalysis, post-translational modification, binding] with the "evidence" data [which include scores for neighborhood, gene fusion, co-occurrence, co-expression, experiments, databases and text mining respectively, and a combined score] of all $C$. acetobutylicum genes for cytoscape analysis [80].

\section{Additional files}

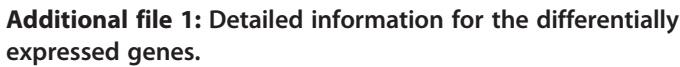

\section{Abbreviations}

TRNs: Transcriptional regulatory networks; TF: Transcription factor; TFBSs: TF binding sites; TR: Transcriptional regulator; DEGs: Differentially expressed genes; RSAT: Regulatory sequence analysis tools; OMA: Orthology MAtrix; TGs: Target genes; PPI: Protein-protein interaction; qRT-PCR: quantitative real time polymerase chain reaction; SAM: Significance analysis of microarrays; MeV: Multiple experiment viewer.

\section{Competing interests}

The authors declare that they have no competing interests.

\section{Authors' contributions}

KPV carried out the fermentations, RNA isolations, microarray hybridization, image processing and data normalization. KPV and QW analyzed the data. QW carried out the regulon analyses and other computational analyses. $\mathrm{HH}$ CHW and ETP helped with data analysis and interpretation, and participated in the manuscript preparation. ETP and CHW conceived the study, participated in its design and coordination. All authors read and approved the final manuscript.

Authors' information

Qinghua Wang and Keerthi Prasad Venkataramanan: co-first author.

\section{Acknowledgements}

The work was supported by the genomic science grant from Department of Energy, USA (grant \# DE-SC0007092). We thank Dr. Shawn Jones for training KPV in fermentation and microarray experiments. We acknowledge members of Papoutsakis laboratory for their assistance during sampling of the cultures for RNA isolation. We thank Chuming Chen, Manabu Torii and Jia Ren at the CBCB for helpful discussions regarding data analyses. And we are grateful to Evert-Jan Blom at University of Groningen, Netherlands for his kind assistance in implementing the software MOTIFATOR.

\section{Author details}

'Delaware Biotechnology Institute, University of Delaware, Newark, DE 19711, USA. ${ }^{2}$ Center for Bioinformatics and Computational Biology, University of Delaware, Newark, DE 19711, USA. ${ }^{3}$ Department of Computer and Information Sciences, University of Delaware, Newark, DE 19711, USA. ${ }^{4}$ Department of Chemical and Biomolecular Engineering, University of Delaware, Newark, DE 19711, USA.

Received: 28 August 2013 Accepted: 30 October 2013

Published: 6 November 2013

\section{References}

1. Van den Abbeele P, et al: Butyrate-producing Clostridium cluster XIVa species specifically colonize mucins in an in vitro gut model. Isme Journal 2013, 7(5):949-961.

2. Tracy BP, et al: Clostridia: the importance of their exceptional substrate and metabolite diversity for biofuel and biorefinery applications. Curr Opin Biotechnol 2012, 23(3):364-381.

3. Fast AG, Papoutsakis ET: Stoichiometric and energetic analyses of nonphotosynthetic $\mathrm{CO}$-fixation pathways to support synthetic biology strategies for production of fuels and chemicals. Curr Opin Chem Eng 2012, 1(4):380-395.

4. Papoutsakis ET: Engineering solventogenic clostridia. Curr Opin Biotechnol 2008, 19(5):420-429.

5. Jones $S W$, et al: The transcriptional program underlying the physiology of clostridial sporulation. Genome Biol 2008, 9(7):R114.

6. Alsaker K, Papoutsakis ET: Microarray-based analysis of the stress response in clostridium acetobutylicum cultures. Abstr Pap Am Chem Soc 2004, 227:U245-U245

7. Alsaker KV, Paredes C, Papoutsakis ET: Metabolite stress and tolerance in the production of biofuels and chemicals: gene-expression-based systems analysis of butanol, butyrate, and acetate stresses in the anaerobe Clostridium acetobutylicum. Biotechnol Bioeng 2010, 105(6):1131-1147.

8. Alsaker KV, Spitzer TR, Papoutsakis ET: Transcriptional analysis of spoOA overexpression in Clostridium acetobutylicum and its effect on the cell's response to butanol stress. J Bacteriol 2004, 186(7):1959-1971.

9. Honicke $\mathrm{D}$, et al: Global transcriptional changes of Clostridium acetobutylicum cultures with increased butanol: acetone ratios. N Biotechnol 2012, 29(4):485-493.

10. Tomas CA, Beamish J, Papoutsakis ET: Transcriptional analysis of butanol stress and tolerance in Clostridium acetobutylicum. J Bacteriol 2004, 186(7):2006-2018.

11. Janssen $\mathrm{H}$, et al: A proteomic and transcriptional view of acidogenic and solventogenic steady-state cells of Clostridium acetobutylicum in a chemostat culture. Appl Microbiol Biotechnol 2010, 87(6):2209-2226.

12. Hou S, et al: Workflow for quantitative proteomic analysis of Clostridium acetobutylicum ATCC 824 using iTRAQ tags. Methods 2013, 61(3):269-276.

13. Emerson JE, et al: Microarray analysis of the transcriptional responses of Clostridium difficile to environmental and antibiotic stress. $J$ Med Microbiol 2008, 57(6):757-764.

14. Yang SH, et al: Clostridium thermocellum ATCC27405 transcriptomic, metabolomic and proteomic profiles after ethanol stress. BMC Genomics 2012, 13:336.

15. Baer SH, Blaschek HP, Smith TL: Effect of butanol challenge and temperature on lipid-composition and membrane fluidity of butanoltolerant clostridium-acetobutylicum. Appl Environ Microbiol 1987, 53(12):2854-2861.

16. Baut $F$, et al: Investigation of acetone-butanol-ethanol fermentation by fluorescence. Appl Microbiol Biotechnol 1994, 41(5):551-555.

17. Isar J, Rangaswamy V: Improved n-butanol production by solvent tolerant Clostridium beijerinckii. Biomass \& Bioenergy 2012, 37:9-15.

18. Brochado AR, Typas A: High-throughput approaches to understanding gene function and mapping network architecture in bacteria. Curr Opin Microbiol 2013, 16(2):199-206.

19. Cho B-K, Palsson B, Zengler K: Deciphering the regulatory codes in bacterial genomes. Biotechnol J 2011, 6(9):1052-1063.

20. Thomas-Chollier M, et al: RSAT 2011: regulatory sequence analysis tools. Nucleic Acids Res 2011, 39(Web Server):W86-W91.

21. Janky R, van Helden J: Evaluation of phylogenetic footprint discovery for predicting bacterial cis-regulatory elements and revealing their evolution. BMC Bioinforma 2008, 9(1):37. 
22. Novichkov PS, et al: RegPrecise: a database of curated genomic inferences of transcriptional regulatory interactions in prokaryotes. Nucleic Acids Res 2009, 38(Database):D111-D118.

23. Kazakov $A E$, et al: RegTransBase-a database of regulatory sequences and interactions in a wide range of prokaryotic genomes. Nucleic Acids Res 2007, 35(Database issue):D407-D412.

24. Munch R, et al: Virtual Footprint and PRODORIC: an integrative framework for regulon prediction in prokaryotes. Bioinformatics 2005, 21(22):4187-4189.

25. Bailey TL, et al: MEME SUITE: tools for motif discovery and searching. Nucleic Acids Res 2009, 37:W202-W208.

26. Altenhoff $A M$, et al: OMA 2011: orthology inference among 1000 complete genomes. Nucleic Acids Res 2011, 39:D289-D294.

27. Tusher VG, Tibshirani R, Chu G: Significance analysis of microarrays applied to the ionizing radiation response. Proc Natl Acad Sci USA 2001, 98(9):5116-5121.

28. Paredes CJ, et al: A general framework for designing and validating oligomer-based DNA microarrays and its application to Clostridium acetobutylicum. App/ Environ Microbiol 2007, 73(14):4631-4638.

29. Blom EJ, et al: FIVA: Functional Information Viewer and Analyzer extracting biological knowledge from transcriptome data of prokaryotes. Bioinformatics 2007, 23(9):1161-1163.

30. Kanehisa $M$, et al: KEGG for integration and interpretation of large-scale molecular data sets. Nucleic Acids Res 2011, 40(D1):D109-D114

31. Uniprot C: Update on activities at the Universal Protein Resource (UniProt) in 2013. Nucleic Acids Res 2012, 41(D1):D43-D47.

32. Janssen $\mathrm{H}$, et al: A transcriptional study of acidogenic chemostat cells of Clostridium acetobutylicum-Solvent stress caused by a transient n-butanol pulse. J Biotechnol 2012, 161(3):354-365.

33. Schwarz KM, et al: A transcriptional study of acidogenic chemostat cells of Clostridium acetobutylicum - Cellular behavior in adaptation to n-butanol. J Biotechnol 2012, 161(3):366-377.

34. Paredes CJ, Alsaker KV, Papoutsakis ET: A comparative genomic view of clostridial sporulation and physiology. Nat Rev Microbiol 2005, 3(12):969-978.

35. Paredes CJ, Rigoutsos I, Papoutsakis ET: Transcriptional organization of the Clostridium acetobutylicum genome. Nucleic Acids Res 2004, 32(6):1973-1981.

36. Paredes CJ, et al: A general framework for designing and validating oligomer-based DNA microarrays and its application to Clostridium acetobutylicum. Appl Environ Microbiol 2007, 73(14):4631-4638.

37. Schneider TD, Stephens RM: Sequence logos - a New Way to display consensus sequences. Nucleic Acids Res 1990, 18(20):6097-6100.

38. Crooks GE, et al: WebLogo: a sequence logo generator. Genome Res 2004 14(6):1188-1190.

39. Bahl $\mathrm{H}$, et al: Expression of heat shock genes in Clostridium acetobutylicum. FEMS Microbiol Rev 1995, 17(3):341-348.

40. Chastanet A, Fert J, Msadek T: Comparative genomics reveal novel heat shock regulatory mechanisms in Staphylococcus aureus and other Gram-positive bacteria. Mol Microbiol 2003, 47(4):1061-1073.

41. Homuth G, Mogk A, Schumann W: Post-transcriptional regulation of the Bacillus subtilis dnaK operon. Mol Microbiol 1999, 32(6):1183-1197.

42. Schumann W: The Bacillus subtilis heat shock stimulon. Cell Stress Chaperones 2003, 8(3):207-217.

43. Hu Y, et al: Transcriptomic and phenotypic analyses suggest a network between the transcriptional regulators $\mathrm{HrcA}$ and sigma(B) in Listeria monocytogenes. Appl Environ Microbiol 2007, 73(24):7981-7991.

44. Beam CE, Saveson CJ, Lovett ST: Role for $\mathrm{radA} / \mathrm{sms}$ in recombination intermediate processing in Escherichia coli. J Bacterio/ 2002, 184(24):6836-6844

45. Bejerano-Sagie $M$, et al: A checkpoint protein that scans the chromosome for damage at the start of sporulation in Bacillus subtilis. Cell 2006, 125(4):679-690.

46. Tao L, Chattoraj P, Biswas I: CtsR Regulation in mcsAB-Deficient GramPositive Bacteria. J Bacteriol 2012, 194(6):1361-1368.

47. Alsaker KV, Papoutsakis ET: Transcriptional program of early sporulation and stationary-phase events in Clostridium acetobutylicum. J Bacterio 2005, 187(20):7103-7118

48. Millat $\mathrm{T}$, et al: A shift in the dominant phenotype governs the $\mathrm{pH}-$ induced metabolic switch of Clostridium acetobutylicumin phosphatelimited continuous cultures. Appl Microbiol Biotechnol 2013,

97(14):6451-6466
49. Foster JW: Escherichia coli acid resistance: tales of an amateur acidophile. Nat Rev Microbio/ 2004, 2(11):898-907.

50. Borden JR, et al: A genomic-library based discovery of a novel, possibly synthetic, acid-tolerance mechanism in Clostridium acetobutylicum involving non-coding RNAs and ribosomal RNA processing. Metab Eng 2010, 12(3):268-281.

51. Broadbent JR, et al: Physiological and transcriptional response of lactobacillus casei ATCC 334 to acid stress. J Bacterio/ 2010,

192(9):2445-2458

52. Trip H, Mulder NL, Lolkema JS: Improved Acid Stress Survival of Lactococcus lactis Expressing the Histidine Decarboxylation Pathway of Streptococcus thermophilus CHCC1524. J Biol Chem 2012, 287(14):11195-11204.

53. Hirasawa $T$, et al: Identification of target genes conferring ethanol stress tolerance to Saccharomyces cerevisiae based on DNA microarray data analysis. J Biotechnol 2007, 131(1):34-44

54. Santiago B, et al: The branched-chain amino acid aminotransferase encoded by ilvE is involved in acid tolerance in Streptococcus mutans. J Bacteriol 2012, 194(8):2010-2019.

55. Foster JW: When protons attack: microbial strategies of acid adaptation Curr Opin Microbiol 1999, 2(2):170-174

56. Leyn SA, et al: Genomic reconstruction of the transcriptional regulatory network in bacillus subtilis. J Bacteriol 2013, 195(11):2463-2473.

57. Michel B: After 30 years of study, the bacterial SOS response still surprises us. Plos Biology 2005, 3(7):e255.

58. Nicolaou SA, Gaida SM, Papoutsakis ET: A comparative view of metabolite and substrate stress and tolerance in microbial bioprocessing: from biofuels and chemicals, to biocatalysis and bioremediation. Metab Eng 2010, 12(4):307-331.

59. Shiba T, et al: Sos-Inducible DNA-Repair Proteins, Ruva and Ruvb, of Escherichia-Coli - Functional Interactions between Ruva and Ruvb for Atp Hydrolysis and Renaturation of the Cruciform Structure in Supercoiled DNA. Proc Natl Acad Sci USA 1991, 88(19):8445-8449.

60. Sancar GB, et al: The uvrB gene of Escherichia coli has both lexArepressed and lexA-independent promoters. Cell 1982, 28(3):523-530.

61. Au N, et al: Genetic composition of the Bacillus subtilis SOS system J Bacteriol 2005, 187(22):7655-7666.

62. Ravcheev DA, et al: Transcriptional regulation of central carbon and energy metabolism in bacteria by redox-responsive repressor Rex. J Bacteriol 2011, 194(5):1145-1157.

63. Wietzke $M, B a h l ~ H$ : The redox-sensing protein Rex, a transcriptional regulator of solventogenesis in Clostridium acetobutylicum. Appl Microbiol Biotechnol 2012, 96(3):749-761.

64. Hillmann F, et al: PerR acts as a switch for oxygen tolerance in the strict anaerobe Clostridium acetobutylicum. Mol Microbiol 2008, 68(4):848-860.

65. Hillmann F, et al: The role of PerR in O2-affected gene expression of clostridium acetobutylicum. J Bacterio/ 2009, 191(19):6082-6093.

66. Makarova KS, Mironov AA, Gelfand MS: Conservation of the binding site for the arginine repressor in all bacterial lineages. Genome Biol 2001, 2(4). RESEARCH0013.

67. Lim D, et al: Nucleotide sequence of the argR gene of Escherichia coli K12 and isolation of its product, the arginine repressor. Proc Natl Acad Sci 1987, 84(19):6697-6701.

68. Schaumburg CS, Tan M: Arginine-dependent gene regulation via the ArgR repressor is species specific in Chlamydia. J Bacteriol 2006, 188(3):919-927.

69. Blom EJ, et al: MOTIFATOR: detection and characterization of regulatory motifs using prokaryote transcriptome data. Bioinformatics 2009, 25(4):550-551.

70. Schneider A, Dessimoz C, Gonnet GH: OMA Browser - Exploring orthologous relations across 352 complete genomes. Bioinformatics 2007, 23(16):2180-2182.

71. Even S, et al: Global control of cysteine metabolism by CymR in Bacillus subtilis. J Bacterio/ 2006, 188(6):2184-2197.

72. Tanous $C$, et al: The CymR regulator in complex with the enzyme CysK controls cysteine metabolism in Bacillus subtilis. J Biol Chem 2008, 283(51):35551-35560.

73. Franceschini $A$, et al: STRING v9.1: protein-protein interaction networks, with increased coverage and integration. Nucleic Acids Res 2013, 41(D1):D808-D815.

74. Venkataramanan KP, et al: The Clostridium small RNome that responds to stress: the paradigm and importance of toxic metabolite stress in C. acetobutylicum. BMC Genomics. In press. 
75. Fu Y, Jarboe LR, Dickerson JA: Reconstructing genome-wide regulatory network of E. coli using transcriptome data and predicted transcription factor activities. BMC Bioinforma 2011, 12(1):233.

76. Smyth GK: Limma: linear models for microarray data. In Bioinformatics and Computational Biology Solutions Using R and Bioconductor. New York: Springer; 2005:397-420.

77. Yang H: A segmental nearest neighbor normalization and gene identification method gives superior results for DNA-array analysis. Proc Natl Acad Sci 2003, 100(3):1122-1127.

78. Saeed Al, et al: TM4: a free, open-source system for microarray data management and analysis. Biotechniques 2003, 34(2):374-378.

79. Eisen $\mathrm{MB}$, et al: Cluster analysis and display of genome-wide expression patterns. Proc Natl Acad Sci USA 1998, 95(25):14863-14868.

80. Shannon P, et al: Cytoscape: A software environment for integrated models of biomolecular interaction networks. Genome Res 2003, 13(11):2498-2504.

doi:10.1186/1752-0509-7-120

Cite this article as: Wang et al: Transcription factors and genetic circuits orchestrating the complex, multilayered response of Clostridium acetobutylicum to butanol and butyrate stress. BMC Systems Biology 2013 7:120

\section{Submit your next manuscript to BioMed Central} and take full advantage of:

- Convenient online submission

- Thorough peer review

- No space constraints or color figure charges

- Immediate publication on acceptance

- Inclusion in PubMed, CAS, Scopus and Google Scholar

- Research which is freely available for redistribution 